\title{
Efficient Packet Delivery for Underwater Acoustic Sensor Networks
}

\author{
Zhi Ren, Jianbo Zhang \\ Chongqing Key Laboratory of Mobile Communication Technology, \\ Chongqing University of Posts and Telecommunications \\ Chongqing, China \\ renzhi@cqupt.edu.cn, 1025083236@qq.com
}

Keywords: Underwater Sensor Networks; Acoustic communica-tions; Geographic Information; Routing ; multi-hop

Abstract. Underwater acoustic sensor networks (UWASN) is an important tool of human to monitor the ocean. However, the existing routing has problem of low packet delivery, the long time end to end delay and the redundant packets. A new routing IGDAR is proposed in this article. The IGDAR select candidate node that the distance to the sink is minimum, and compose a candidate forwarder set. Theoretical analysis verifies the effective of IGDAR. Simulation results show that IGDAR increase the packet delivery by $54.3 \%$, reduce the end to end delay and redundant packet by $2.5 \%$ and $12.5 \%$, respectively.

\section{INTRODUCTION}

Underwater Acoustic Sensor Networks (UW-ASNs) consist of devices with sensing, processing, and communication capabilities that are random deployed to perform collaborative monitoring tasks in a given body of water[1][2]. UW-ASNs are usually used to support applications for oceanographic data collection[3], offshore exploration, disaster prevention, assisted navigation[4], distributed tactical surveillance, and mine reconnaissance.

As we know the geographic routing is a hot spot in the UW-ASNs[5]. The main objective of this paper is propose a new geographic routing IGDAR to address the low packet delivery, long time end to end delay and redundant packet, which is faced by GEDAR and other geographic routings[6]. In this article we assumed all nodes dynamic and know the position of the sink node and themselves'.

The rest of this paper is structured as follows. Section (II) describes are related works of this study, Section (III) provides a detail introduction of IGDAR, Section(IV) shows the performance of IGDAR, Section(V) presents the final results and future work.

\section{RELATED WORK}

VBF(Vector-Based Forwarding) is the first routing protocol proposed for the underwater sensor networks[7]. In VBF routing the path transmission is specified by a vector from a sender to a destination, and this vector is located in the center of a pipe routing, the entire nodes in this pipe are candidate for packet transmission. When a node receives a packet, it firstly calculates its position, if the node determines that it is included in the pipe, it continue transmission of the packet transmission otherwise it discard the packet. To saving energy consumption, the selection of eligible node for packet forwarding is determinate with a desirableness factor which is defined as :

$$
\alpha=\frac{\mathrm{p}}{w}+\frac{(R-d \times \cos \theta)}{R}
$$

Where $\mathrm{p}$ is the projection of $\mathrm{A}$ to the routing pipe, $\mathrm{d}$ is the distance between the candidate forwarding node $\mathrm{A}$ and the current forwarding node F. $\theta$ is the angle between the vector $\overrightarrow{F S_{0}}$ and $\overrightarrow{F A}, \mathrm{R}$ is the transmission range, $\mathrm{W}$ is the radius of the route pipe. 


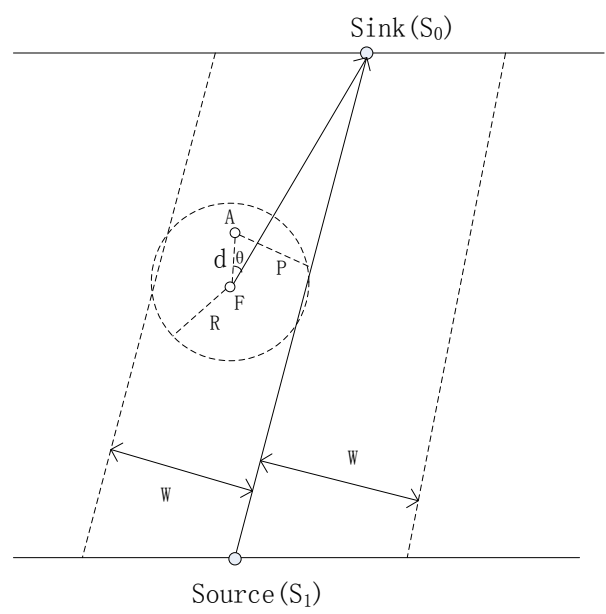

Figure 1. VBF routing protocol

The performance of VBF protocol can be decreased on account of two fundamental problems: the sensitivity to the routing pipe's radius and the second is low delivery ratio in sparse networks. To overcome the drawbacks of VBF, the hop by hop VBF (HH-VBF)[8] has been proposed based on VBF. In VBF routing protocol just only pipe is created from the source to the sink, however, in HHVBF at each hop a virtual pipe is created, so a hop-by-hop approach is used in the routing operation.

DBR(Depth-Based Routing)[9]routing is the first underwater sensor network routing protocol that uses node depth information to transmission data packets. The basic idea of DBR is to forward data packets greedily towards the water surface. During the transmission, the current forward node broadcasts the packet. After receiving the packets, if the node's depth is lower than the forwarder then broadcasts the packets, otherwise just discard the packet.

GEDAR (Geographic and opportunistic routing with Depth Adjustment-based topology control for communication Recovery over void regions)[10] GEDAR use the location information of neighbor nodes and some known sonobuoys to select a next-hop forwarder set from neighbors to continue forwarding the packet until to the destination. To avoid unnecessary transmissions, low priority nodes suppress their transmissions whenever they detect that the same packet was sent by a high priority node. Through in-depth study, we found that in GEDAR when select the next-hop forward from the set of neighbors by the distance multiply the delivery probability may be decrease by the distance from the forwarding to the sink nodes. Then we propose the IGDAR (Improved GEDAR) routing protocol.

In the known geographic routings the general problem is the end to end delay and the low packet delivery. Then we proposed the IGDAR routing to solve the problem.

\section{IGDAR ROUTING}

In order to as soon as possible to send packet from the forwarder node to the sink. The IGDAR contain the following new mechanism.

\section{A. new mechanism of IGDAR}

To decrease the redundant number of packets in the network forwarding:

1) The first new mechanism: the forwarder node select the node is closer to the sink than the forwarder node in the transmission radius, then, make up a forwarder candidate set. The optimal node which is select from the set.

2) The second new mechanism: the forwarder choose the candidate node whose distance to the forwarder and the distance to the sink is the minimum among the neighbor nodes. This new method can decrease the probability of packet collision, then add its delivery to the sink node.

3) the third new mechanism: when the forwarder has select the candidate forwarder set to transmission the packet, the forwarder use the nodes ID instead the nodes geographic information, to inform the selected nodes, 


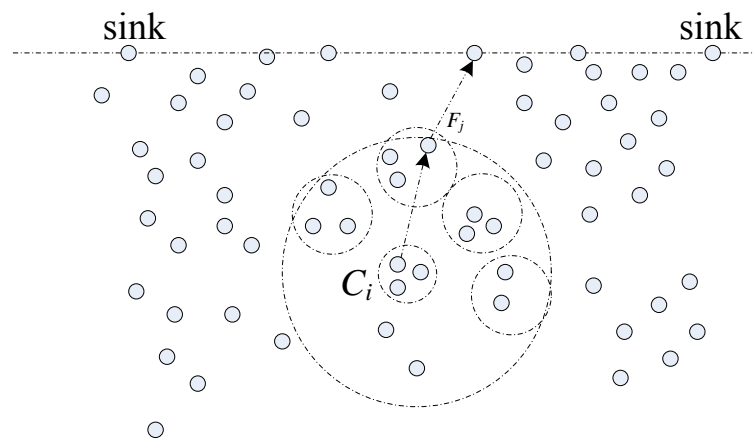

Figure 2. IGDAR routing protocol

\section{B. the operation of IGDAR}

The IGDAR contain the following operation:

1) periodic beaconing

The IGDAR routing use periodic beaconing to update the position message to the underwater nodes. In the beacon messages, each sink node embeds a sequence number, its unique ID, and its $\mathrm{X}, \mathrm{Y}$ location, the depth message since the sink node are deployed on the surface and the vertical movement is negligible with respect to the horizontal movement.

2) forwarder candidate set $C_{i}$ :

When a node has a packet to send, the node decide the candidate set compose by the nodes that is closer to the sink than the forwarder

3) the next forwarder set $F_{j}$ :

For each transmission, the forwarder calculate the candidate node distance to the forwarder and the distance to the sink. The small the value, the higher the priority. Each forwarder set starts with the greatest priority node in the $C_{i}$ and is expanded by including the nodes which the distance is less than $\frac{1}{2} r_{c}$ (the node transmission radius), then each $F_{j}$ is expanded to included those nodes in $C_{i}$ that has a distance of less than the communication $r_{c}$ for all nodes already in the cluster. After the calculation, the forwarder select the highest priority set as the next-hop forwarder set. the rest forwarder set just transmission the packets by order only if the highest priority set fails to transmission.

4)the transmisson process: the node which receive the packet repeat the step 1), 2), 3), until the packet delivery to the sink.

\section{C. performance analysis}

In the GEDAR and IGDAR routings, from the same resource use different routing to transmission the sink. The GEDAR consider the probability at a distance of data, which restrict the forwarder select the nearest node to the sink compare with the IGDAR, so when use the two method:

While use the same time the delivery is:

$$
\text { end_to_end_delay } I_{I G D A R}<\text { end_to_end_delay } y_{G E D A R}
$$

$$
\text { Delivery }_{I G D A R}>\text { Delivery }_{G E D A R}
$$

\section{SIMULATION ANALYSIS}

In this part, we simulation the IGDAR routing on OPNET[11] to evaluate the performance of IGDAR and the comparison among DBR, GEDAR about the transmission, the end to end delay and the redundant packets. 
TABLE I. TABLE I. SIMULATION PARAMETERS

\begin{tabular}{cc}
\hline PARAMETERS & VALUE \\
\hline nodes & $150 \sim 450$ \\
region size & $1500 \mathrm{~m} \times 1500 \mathrm{~m} \times$ \\
& $1500 \mathrm{~m}$ \\
data rate & $50 \mathrm{kbps}$ \\
transmission range $\left(\mathrm{r}_{\mathrm{c}}\right)$ & $250 \mathrm{~m}$ \\
nodes speed & $0.3 \mathrm{~m} / \mathrm{s}$ \\
\hline
\end{tabular}

A. Simulation Results

1) Fig.3 shows the results of packets delivery ratio.

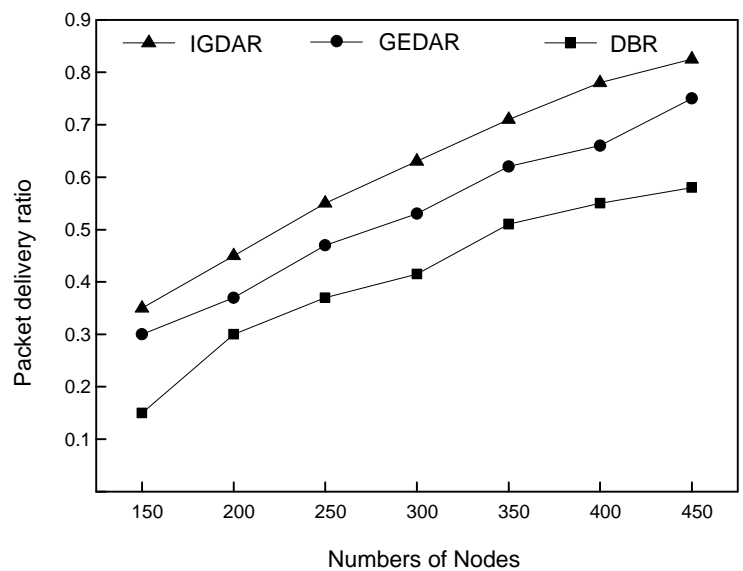

Figure 3. packet delivery ratio

As shown in the Fig.3. We obtain that the packet delivery ratio of IGDAR is more than GEDAR at least $54.3 \%$, also is much better than DBR. Because of the IGDAR decrease the time to select the next hop, the forwarder can use time to forwarding the packets, avoid the time to retransmission the packets by other candidate node.

2) Fig.4 shows the end to end delay

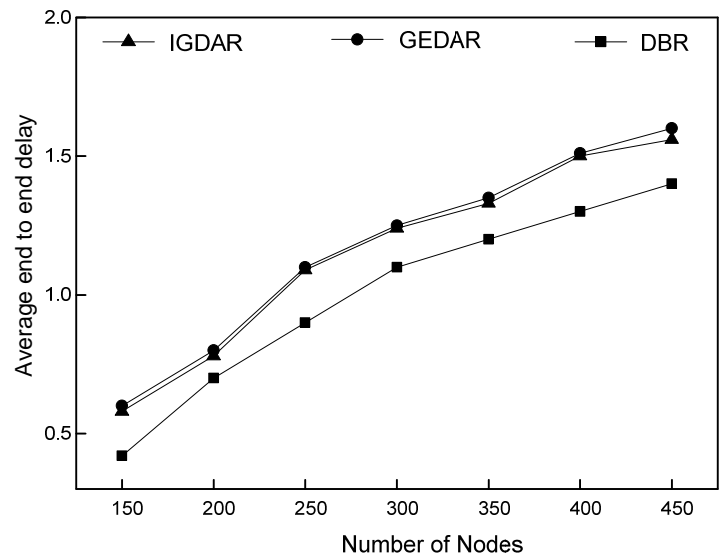

Figure 4. average end to end delay

As shown in Fig. 4, we know that the end to end delay of IGDAR is lower than GEDAR and DBR at least $2.5 \%$, and much better than DBR. This is because the IGDAR routing transmission select the optimal node by the closest to the sink node. It proved that the IGDAR performance is better than GEDAR. 
3) Fig.5 show the results of redundant packets

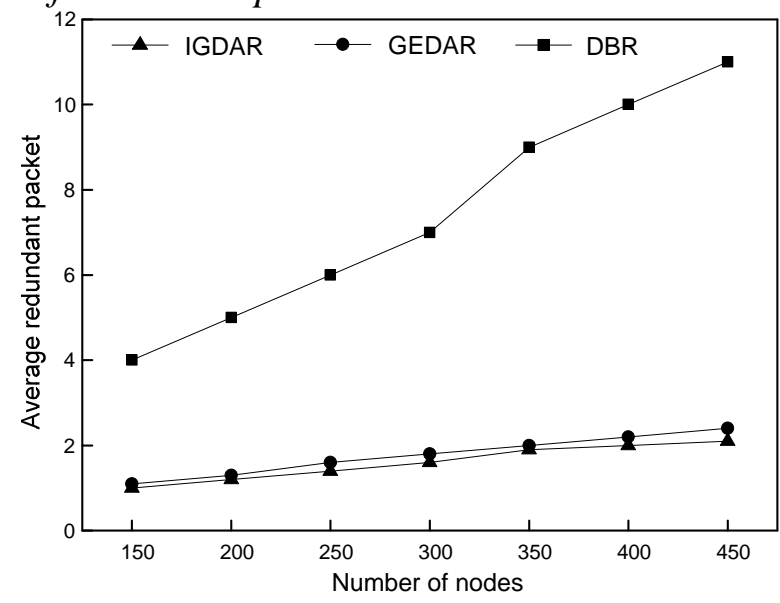

Figure 5. average number of redundant packet

As shown in Fig.5 the number of redundant packets is increase in DBR routing when the network density increases, the problem is caused by DBR broadcasts packets. However, in GEDAR and IGDAR, the both routings are just select a few of nodes as the candidate nodes to delivery the packets. The results show that the IGDAR is save about $12.5 \%$ average redundant packets.

\section{CONCLUSION}

This work proposes a routing based on node geographic information for underwater acoustic networks. IGDAR efficiently increase the packet delivery by $54.3 \%$, reduce the end to end delay and redundant packet by $2.5 \%$ and $12.5 \%$, respectively. So it proved works well.

As future work, we plan to apply this routing to solve the node void region, and increase the network lifetime.

\section{ACKNOWLEDGMENT}

This work is sponsored by The National Natural Science Foundation of China(61379159), Chongqing education projections(Kizh11206).

\section{REFERENCES}

[1] Dario Pompili, Tommaso Melodia, Ian F. Akyiliz, "Distributed ro-uting algorithm for underwater acoustic sensor networks," IEEE transactions on wireless communications, vol. 9, no.9, pp.2934-2944, Sep. 2010.

[2] A. Gkikopouli, G. Nikolakopoulos, S.Manesis, "a survey on under-water wireless sensor networks and applications," 201220 th Mediterranean conference on control and automation, 2012, pp.1147-1154.

[3] Muhammad Ayaz, Imran Baig, Azween Abdullah, "a survey on routing techniques in underwater wireless sensor networks," journal of network and computer applications, 2011, pp.1980-1927.

[4] Tariq Ali, Low Tang Jung, Ibrahima Faye, "classification of routin-g algorithms in volatile environment of underwater wireless sensor networks," international journal of communication networks and information security, 2014,vol.6, no.2,pp.129-147.

[5] Muhammad Ayaz, Azween Abdullah, Ibrahima Faye, "an efficient dynamic addressing based routing protocol for underwater wireless sensor networks," computer communications, 2012,pp.475-486.

[6] Li Juelong, Du Xiaofei, Xing Jianchun, "location based adaptive r-outing protocol for underwater acoustic sensor networks," Automatic C-ontrol and Artificial Intelligence (ACAI 2012), International Conference on,2012, pp.1315-1319, doi: 10.1049/cp.2012.1221 
[7] Wang Ping, Fu Donghao, Xing Jianchun, "a reliable and efficient r-outing protocol for underwater acoustic sensor networks," IEEE 2013 in-ternational conference on Cyber Technology in Automation, Control and Intelligent Systems, 2013,pp.185-190,doi: 10.1109/CYBER.2013.6705443.

[8] Pen Xie, Zhong Zhou, Nicolas Nicolaou, "efficient vector-based f-orwarding for underwater sensor networks," Hindawi Publishing Corpor-ation, 2010, pp.1-13.

[9] Masoumeh Slimohammmadi, Shahpour. Alirezaee, Mahmood Ah-madi, "Distance based underwater routing protocol DBURP," internatio-nal journal of computer science and network solutions, 2014, vol.2, no.4, pp.37-46.

[10] Rodolfo W.L. Coutinho, Azzedine Boukerche, Luiz F.M. Vieira, "geographic and opportunistic routing for underwater sensor networks," I-EEE Transactions on Computers, vol. 65, no.2, 2016, pp.548-561.

[11] Riverbed modeler[EB/OL]. http://www.riverbed.com/products/per-formance-m-anagementcontrol/network-performance-management/network-simulation.html,2015. 